\title{
MICROESTRUTURA, MICRODUREZA E PARÂMETROS TÉRMICOS DA LIGA DE SOLDA Sn-40\%Bi-2\%Ag*
}

\author{
Vítor Covre Evangelista da Silva \\ José Eduardo Spinelli ${ }^{2}$ \\ Bismarck Luiz Silva ${ }^{3}$
}

\begin{abstract}
Resumo
Esta proposta objetivou desenvolver uma análise teórico/experimental da influência de $2 \%$ $\mathrm{Ag}$ (em peso) em uma liga de solda $\mathrm{Sn}-40 \% \mathrm{Bi}$ solidificada unidirecionalmente em regime transitório de fluxo de calor. Correlações experimentais entre os parâmetros térmicos de solidificação como velocidade de deslocamento da isoterma liquidus - $\mathrm{V}_{\mathrm{L}}$, taxa de resfriamento - $\dot{T}_{L}$ e parâmetros microestruturais como espaçamentos dendríticos secundários $-\lambda_{2}$ foram determinados. A caracterização microestrutural foi realizada por meio de microscopia ótica e eletrônica, difração de raios- $x$, além de fluorescência de raios- $x$ para obtenção dos perfis de macrossegregação dos solutos ( $\mathrm{Bi}, \mathrm{Ag})$. Para determinação do perfil de dureza foram realizados ensaios de dureza Vickers. As microestruturas brutas de fusão da liga Sn-40\%Bi-2\%Ag são compostas de dendritas ricas em Sn decoradas com partículas de $\mathrm{Bi}$ circundadas por uma mistura eutética $(\mathrm{Sn}+\mathrm{Bi})$ e partículas primárias de $\mathrm{Ag}_{3} \mathrm{Sn}$. Os parâmetros térmicos decresceram com aumento da camada solidificada, permitindo uma ampla variação: $\dot{T}_{L} \rightarrow 0,1$ a $10,0^{\circ} \mathrm{C} / \mathrm{s}, V_{L} \rightarrow 0,2$ a $0,9 \mathrm{~mm} / \mathrm{s}$ e $\mathrm{G}_{\mathrm{L}} \rightarrow 0,4$ a $11,0^{\circ} \mathrm{C} / \mathrm{mm}$. O teor de bismuto não variou significativamente, ao passo que a prata variou de forma efetiva, concentrando-se no início do lingote (até próximo de $35 \mathrm{~mm}$ ). O surgimento de braços dendríticos terciários em $15 \mathrm{~mm}$ conteve a diminuição da microdureza Vickers, esperada para as posições mais afastadas da interface metal/molde.
\end{abstract}

Palavras-chave: Ligas de soldagem Sn-Bi; Solidificação; Dureza.

\section{MICROSTRUCTURE, MICROHARDNESS AND THERMAL PARAMETERS OF Sn-40\%Bi-2\%Ag SOLDER ALLOY}

\section{Abstract}

This study aimed to develop a theoretical/experimental analysis of the influence of silver addition $(2 \% \mathrm{Ag})$ in the $\mathrm{Sn}-40 \mathrm{wt} \% \mathrm{Bi}$ solder solidified directionally under unsteady-state conditions. Experimental correlations between thermal parameters as tip growth rate $-V_{L}$, cooling rate - $\dot{T}_{L}$ and microstructural parameters as secondary dendritic arm were determined. The microstructural characterization was carried out by light and electronic microscopy, x-ray diffraction, further of flurescence spectrometer to obtain the macrossegregation profiles of solutes $(\mathrm{Bi}, \mathrm{Ag})$. A hardness Vickers tester was used in order to determine the hardness profile of the Sn40wt\%-2wt\%Ag solder. The as-cast microstructures of $\mathrm{Sn}-\mathrm{Bi}-\mathrm{Ag}$ alloy were arranged by $\mathrm{Sn}$-rich dendrites decorated with $\mathrm{Bi}$ precipitates in their own core surrounded with a eutectic mixture (Bi-rich and Sn-rich phases) and primary $\mathrm{Ag}_{3} \mathrm{Sn}$ intermetallic particles. The thermal parameters decreased with increase of the solidified layer, allowing a wide range: $\dot{T}_{L} \rightarrow 0.1-10.0^{\circ} \mathrm{C} / \mathrm{s}, V_{L} \rightarrow 0.2 \mathrm{~mm}-0.9 \mathrm{~mm} / \mathrm{s}$ and $\mathrm{G}_{\mathrm{L}} \rightarrow 0.4-11.0^{\circ} \mathrm{C} / \mathrm{mm}$. The Bi content did not changed significantly, whereas Ag content has changed effectively, mainly at the beginning of the ingot (close to $35 \mathrm{~mm}$ ). The growth tertiary from $15 \mathrm{~mm}$ contained the decrease of the hardness values, expected for the furthest positions of the metal/mold interface.

Keywords: Sn-Bi solder alloys; Solidification; Hardness.

Engenharia de Materiais, graduação, iniciação científica, Departamento de Engenharia de Materiais, Universidade Federal de São Carlos, São Carlos, São Paulo e Brasil.

2 Engenharia Mecânica, pós-doc, orientador, Departamento de Engenharia de Materiais, Universidade Federal de São Carlos, São Carlos, São Paulo e Brasil.

3 Engenharia de Materiais, doutorando, Departamento de Engenharia de Materiais, Universidade Federal de São Carlos, São Carlos, São Paulo e Brasil. 


\section{INTRODUÇÃO}

O processo de soldagem tem um papel fundamental na fabricação de produtos eletrônicos desde o inicio da era. A conexão de produtos eletrônicos baseia-se na solidificação de um metal de adição. As ligas Sn-37\%Pb e Sn-40\%Pb consolidaramse como ligas de ótimas propriedades térmicas, mecânicas, físicas e químicas para aplicações da indústria eletrônica [1,2]. Porém, o chumbo é tóxico, tendo papel prejudicial à saúde dos seres humanos e ao meio ambiente. Desse modo, diversas legislações foram criadas em diferentes lugares do mundo para amenizar e até proibir a adição de $\mathrm{Pb}$ em componentes eletrônicos. Desde então, apesar de resistências por parte do setor empresarial e político, indústrias e centros de pesquisa em todo o mundo passaram a desenvolver novas ligas de solda livres de chumbo (Lead-free solder alloys). Segundo Abtew e Selvaduray [1] mais de 70 sistemas metálicos estão sendo continuamente estudados, sejam eles binários, ternários ou quaternários. Dentre esses novos sistemas, a adição de $\mathrm{Ag}$ em ligas Sn-Bi é apontada na literatura como uma possibilidade para substituir as tradicionais ligas $\mathrm{Sn}-\mathrm{Pb}$ em processos de brasagem [3]. Estas melhorias, por sua vez, estão fortemente ligadas às características microestruturais formadas durante a solidificação.

O presente trabalho objetivou desenvolver uma análise teórico/experimental da influência da adição de $2 \% \mathrm{Ag}$ (em peso) em uma liga hipoeutética $\mathrm{Sn}-40 \% \mathrm{Bi}$ solidificada unidirecionalmente em regime transitório de fluxo de calor - simulação do processo industrial. A liga $\mathrm{Sn}-40 \% \mathrm{Bi}-2 \% \mathrm{Ag}$ foi avaliada quanto ao seu desenvolvimento microestrutural e dureza com suas respectivas correlações com os parâmetros térmicos de solidificação: velocidade da isoterma liquidus $\left(\mathrm{V}_{\mathrm{L}}\right)$ e taxa de resfriamento $\left(\dot{T}_{\mathrm{L}}\right)$. A caracterização microestrutural foi realizada através das técnicas de microscopia ótica e eletrônica (com a medição dos espaçamentos dendríticos, $\lambda_{1}$, $\left.\lambda_{2}, \lambda_{3}\right)$, difração de raios-x e fluorescência de raios-x para obter os perfis de macrossegregação dos solutos ao longo do fundido. Correlações experimentais do tipo parâmetros térmicos/parâmetros estruturais e dureza/parâmetros estruturais são discutidas.

\section{MATERIAIS E MÉTODOS}

Para realização dos experimentos de solidificação direcional da liga $\mathrm{Sn}-40 \% \mathrm{Bi}-2 \% \mathrm{Ag}$ utilizou-se um dispositivo de solidificação unidirecional vertical ascendente, detalhado por Rosa et al [4] e Canté e colaboradores [5]. O dispositivo é composto por um sistema de aquecimento (resistências elétricas envolvidas por uma casca cilíndrica refratária) e por um conjunto lingoteira/chapa molde (aço carbono 1020) que são responsáveis pela fusão e solidificação do metal líquido, respectivamente.

O lingote fundido foi seccionado na direção longitudinal e depois transversalmente para obtenção das amostras de diversas posições ao longo do mesmo. As posições foram $5 \mathrm{~mm}, 10 \mathrm{~mm}, 15 \mathrm{~mm}, 20 \mathrm{~mm}, 25 \mathrm{~mm}, 30 \mathrm{~mm}, 40 \mathrm{~mm}, 50 \mathrm{~mm}, 70 \mathrm{~mm}$ e $90 \mathrm{~mm}$ a partir da base refrigerada. As amostras foram lixadas sequencialmente por lixas de 
granulometria 150, 240, 320, 400, 500, 600, 1200 e 1500 mesh em lixadeiras manuais com refrigeração a água. Após lixadas e limpas, elas foram polidas com abrasivo, uma solução de alumina de $1 \mu \mathrm{m}$. Esta etapa foi realizada em politrizes rotativas, tornando assim a superfície a ser analisada pronta para o ataque químico. Por fim, as amostras foram atacadas quimicamente para revelação das microestruturas presentes no fundido. O reagente químico usado foi uma solução de $100 \mathrm{~mL}$ de $\mathrm{H}_{2} \mathrm{O}, 10 \mathrm{~g}$ de $\mathrm{FeCl}_{3}$ e $5 \mathrm{~mL}$ de $\mathrm{HCl}$. Um conjunto de microestruturas (transversais e longitudinais) foi registrado por meio da utilização do microscópio ótico Olympus BX41M-LED e do sistema de processamento de imagens Infinity Capture acoplado ao microscópio.

As medidas de espaçamentos dendríticos primários $\left(\lambda_{1}\right)$ foram realizadas segundo 0 método do triângulo [6], uma vez que as condições de solidificação com fluxo de calor transitório podem proporcionar um crescimento bastante irregular das dendritas. Para quantificar os espaçamentos dendríticos secundários $\left(\lambda_{2}\right)$ e terciários $\left(\lambda_{3}\right)$ foi utilizado o método do intercepto [7]. As medições de $\lambda_{1} / \lambda_{3}$ e $\lambda_{2}$ foram realizadas sobre a secção transversal (perpendicular à direção de extração de calor) e longitudinal do lingote $\mathrm{Sn}-40 \% \mathrm{Bi}-2 \% \mathrm{Ag}$, respectivamente. Foram realizadas, em média, 40 medidas de espaçamento para cada posição analisada.

O durômetro utilizado nos ensaios de dureza Vickers (HV) é da marca Shimadzu modelo HMV-G 20ST. As medidas de dureza (penetrador do tipo Vickers de diamante piramidal de base quadrada e carga de $500 \mathrm{~g}$ ) foram feitas de acordo com a norma ASTM E384-11. Durante os ensaios, tomou-se o cuidado de cumprir o espaçamento mínimo necessário entre as identações e entre cada identação e a borda (2,5d, onde "d" é a diagonal média da identação Vickers) [8]. Para cada posição selecionada ao longo do lingote fundido foram realizadas cerca de 10 medidas de dureza, totalizando 90 valores de HV.

As análises de flourescência de raios-X (FRX) foram realizadas em diversas posições ao longo do lingote $\mathrm{Sn}-40 \% \mathrm{Bi}-2 \% \mathrm{Ag}$. As superfícies foram lixadas até uma granulação de 1200 mesh. O equipamento usado foi um EDX-720 da Shimadzu (LCE/DEMa).

\section{RESULTADOS E DISCUSSÃO}

Foi feito um experimento prévio com o intuito de estabelecer as temperaturas liquidus e eutética da liga. Para isso, vazou-se uma fração do material fundido em um cadinho de carbeto de silício $(\mathrm{SiC})$ recoberto com cimento refratário. Fazendo uso da Regra das Fases de Gibbs, pode-se, pelas variações do comportamento da curva de resfriamento, indicar a variação da quantidade de estados no recipiente. As temperaturas em que ocorreram tais mudanças indicaram os valores da temperatura liquidus e da temperatura eutética, sendo esses valores de $161,2^{\circ} \mathrm{C}$ e $132,5^{\circ} \mathrm{C}$, respectivamente. A curva de resfriamento determinada segue abaixo na Figura 1a.

Os perfis térmicos de solidificação obtidos pelos termopares (tipo J) estão apresentados a seguir na Figura 1b. Esses resultados foram usados como base para a determinação dos parâmetros cinéticos de solidificação, quais sejam taxa de 
resfriamento e velocidade de deslocamento da isoterma liquidus. Indicou-se no gráfico a linha liquidus determinada no experimento prévio. A isoterma liquidus, que indica a mudança no número de fases pela variação de comportamento, também denota a passagem da interface sólido/líquido no ponto, ou em cada posição monitorada.

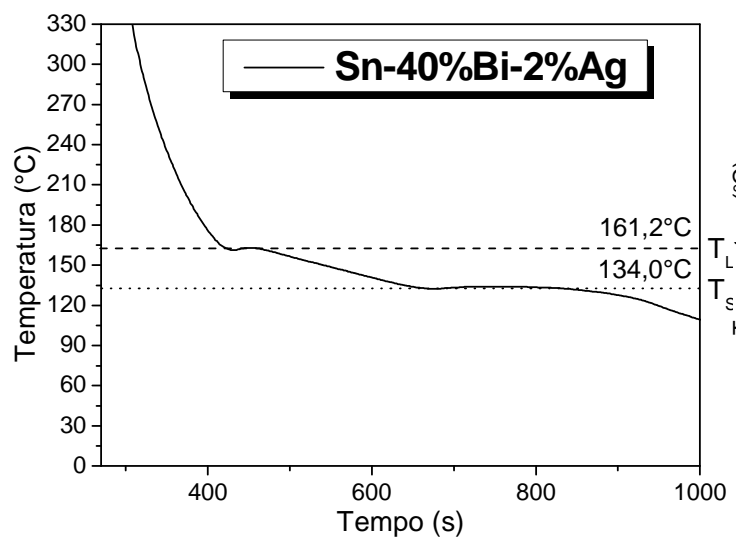

(a)

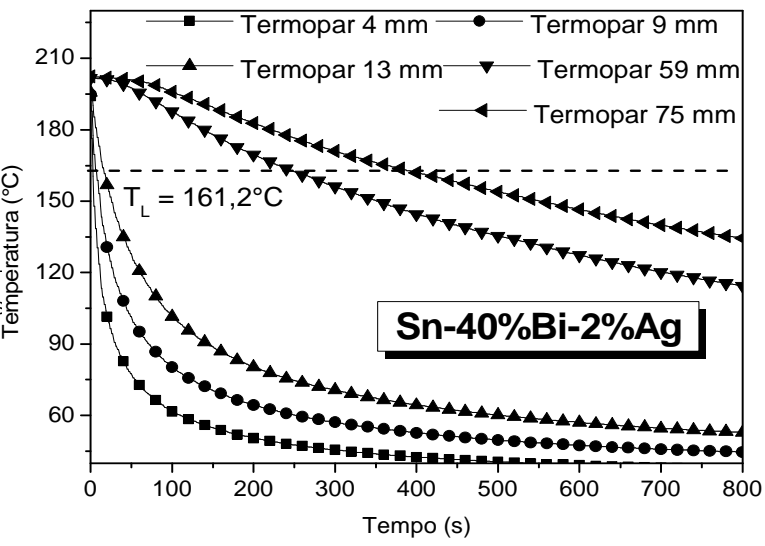

(b)

Figura 1. (a) Curva de resfriamento obtida em experimento prévio e (b) perfis térmicos (temperatura $x$ tempo) determinados pelos termopares, conforme a posição, durante a solidificação direcional ascendente da liga $\mathrm{Sn}-40 \% \mathrm{Bi}-2 \% \mathrm{Ag}$.

As leituras dos termopares foram utilizadas para gerar o gráfico relacionando a passagem da frente liquidus por posição do termopar $(\mathrm{P})$ no lingote, em função do tempo (t). Tais dados encontram-se na Figura 2a. Fazendo-se o ajuste para curva nos pontos experimentais, estabelece-se uma função de potência que relaciona a posição com o tempo. Essa função apresenta forma $P=a(t){ }^{b}$. Derivando-se a função $\mathrm{P}$ em relação ao tempo, determinam-se as velocidades de deslocamento da isoterma liquidus $\left(\mathrm{V}_{\mathrm{L}}\right)$ (Figura 2b). Calculou-se ainda a taxa de resfriamento $\left(\dot{T}_{\mathrm{L}}\right)$ considerando-se a inclinação da curva de resfriamento nos instantes antes e após a passagem da frente liquidus por cada termopar. Na Figura $2 c$ encontram-se os valores da taxa de resfriamento conforme a posição. Os gradientes de temperatura $\left(G_{L}\right)$ foram obtidos a partir da velocidade e taxa de resfriamento pela relação $G_{L}=\frac{i_{L}}{V_{L}}$, exibidos na Figura 2d. Nota-se, pelos resultados, que o valor dos parâmetros térmicos avaliados tende a diminuir conforme se distancia da interface metal $/ \mathrm{molde}$. Isso ocorre devido ao metal solidificado formar uma barreira térmica entre a fonte de extração de calor e o líquido. Esse fato justifica o tipo do experimento de solidificação realizado, o qual envolve fluxo transitório de calor. A variação do fluxo de calor tende a tornar a microestrutura próxima a chapa molde mais fina, enquanto as mais distantes tendem a ser mais grosseiras. 


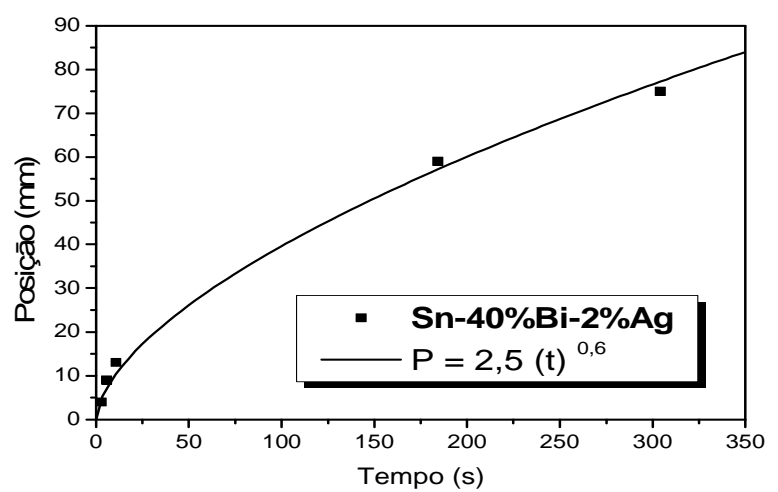

(a)

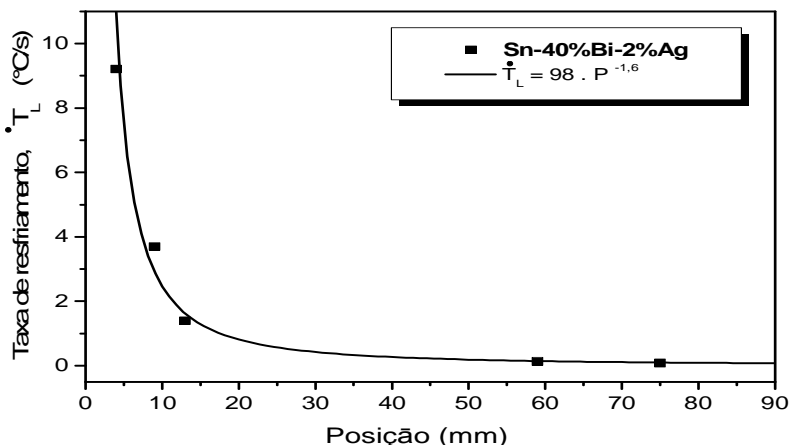

(c)

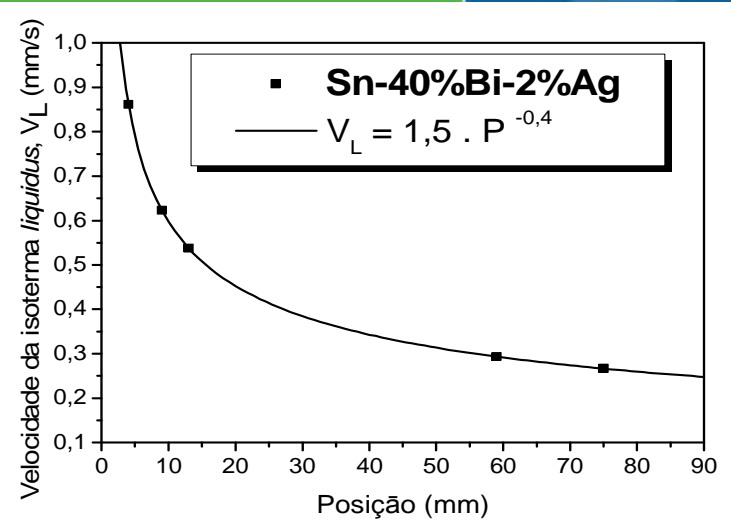

(b)

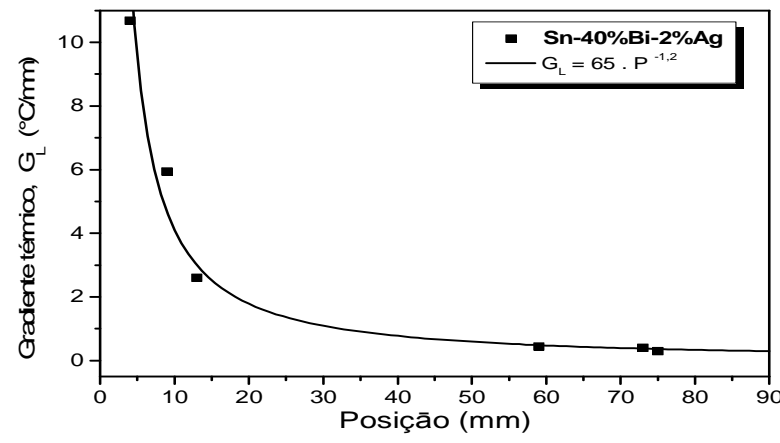

(d)

Figura 2. (a) Posição da isoterma liquidus a partir da interface metal/molde; (b) evolução do avanço da isoterma liquidus $\left(\mathrm{V}_{\mathrm{L}}\right)$; (c) evolução da taxa de resfriamento $\left(\dot{T}_{\mathrm{L}}\right)$ e $(\mathrm{d})$ progresso do gradiente térmico $\left(G_{\llcorner}\right)$com a posição para a liga $\mathrm{Sn}-40 \% \mathrm{Bi}-2 \% \mathrm{Ag}$ solidificada unidirecionalmente.

A macroestrutura obtida teve predominância de grãos colunares e crescimento dendrítico ao longo do lingote (Figura 3). Nota-se que até próximo de $35 \mathrm{~mm}$ houve grande precipitação do intermetálico tipo $\mathrm{Ag}_{3} \mathrm{Sn}$. Este fato está ligado ao elevado teor de prata nas posições iniciais no lingote como visto na Figura $4 b$, a qual tem sua segregação dificultada na interface de sólido/líquido devido sua maior densidade na fase líquida [9]. A posição onde há mudança no teor de prata (cerca de $35 \mathrm{~mm}$ ), marca também uma transição na morfologia do intermetálico $\mathrm{Ag}_{3} \mathrm{Sn}$. Um estudo feito por Garcia e colaboradores [10] analisou a morfologia do intermetálico, que se

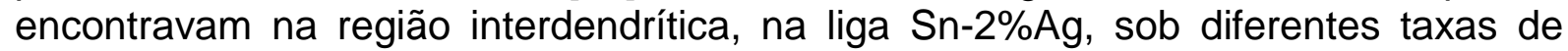
resfriamento, encontrando três tipos de morfologias: esferoides, placas e fibras. No presente estudo, os intermetálicos apresentaram morfologia de placas, no inicio, sofrendo uma transição para semelhantes a placas degeneradas. Os intermetálicos não se concentraram apenas na região interdendrítica, diferentemente do encontrado por Garcia, e apresentaram-se como a fase de formação primária no lingote. Essa mudança de comportamento do intermetálico está relacionada com a diferença de composição entre as ligas. Estudos sobre as ligas $\mathrm{Sn}-\mathrm{Ag}$ e $\mathrm{Sn}-\mathrm{Ag}-\mathrm{Cu}$ relatam que o tamanho, morfologia e a distribuição das partículas de $\mathrm{Ag}_{3} \mathrm{Sn}$ têm um papel importante no comportamento mecânico dessas ligas de solda [10,11].

As dendritas, ricas em estanho, mostram-se em grande quantidade na fase inicial. Conforme se distancia da fonte de extração de calor, a microestrutura vai se tornando mais grosseira e diminui-se o número de dendritas. Esse crescimento e aumento do espaçamento interdendrítico tendem a se estabilizar com o crescimento dos braços dendríticos terciários. $\mathrm{O}$ crescimento desses limita o engrossamento dos braços primários e secundários, devido à disputa que se tem por estanho na 
solução. A posição que evidenciou o primeiro aparecimento de braços terciários foi a $15 \mathrm{~mm}$ da interface metal/molde, que pode ser visto na Figura 3. Este surgimento influencia, também, na distribuição de soluto e das fases secundárias, trazendo uma consequência positiva para as propriedades mecânicas.

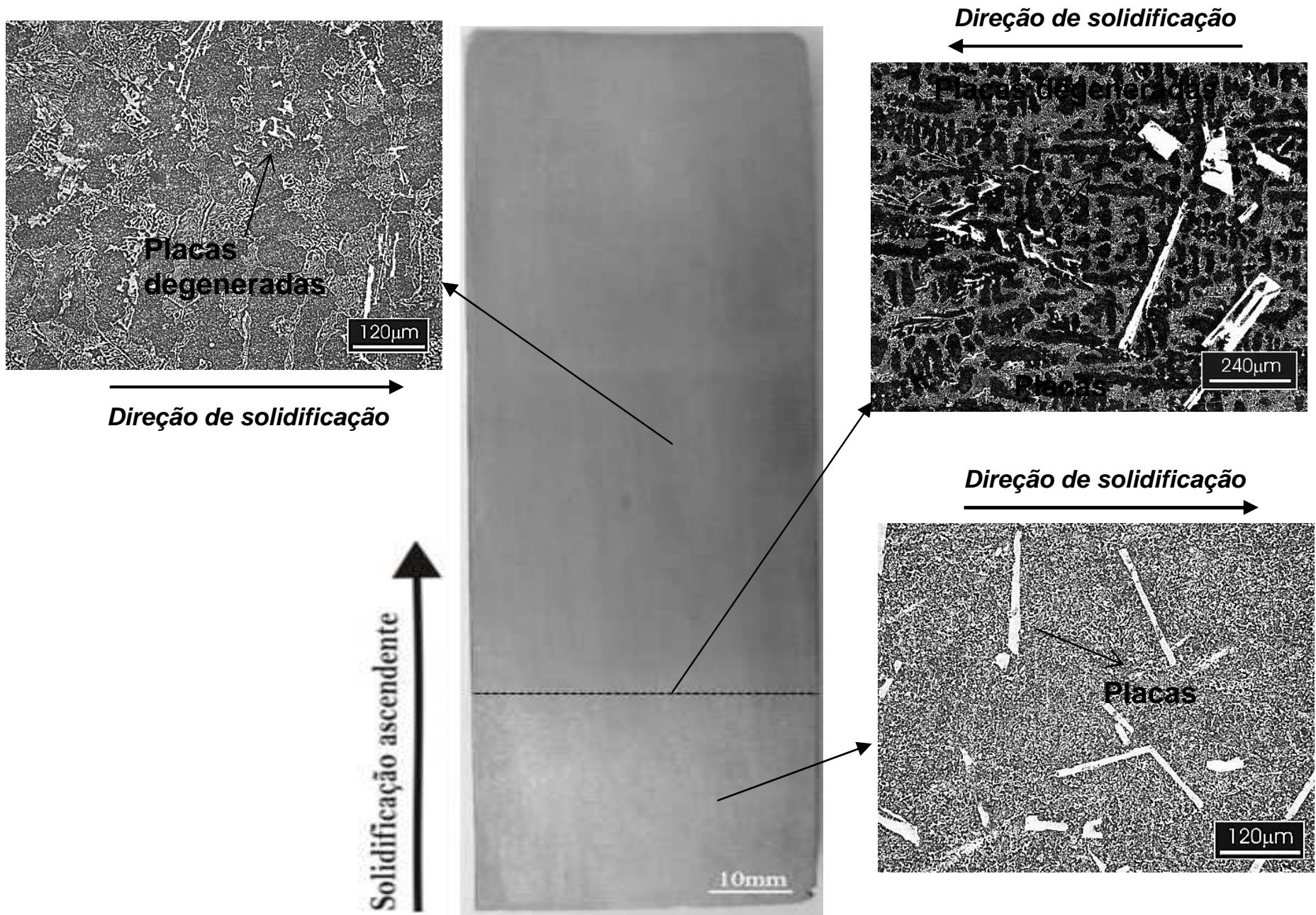

Figura 3. Macroestrutura e microestruturas do lingote $\mathrm{Sn}-40 \% \mathrm{Bi}-2 \% \mathrm{Ag}$. A linha pontilhada indica 0 limite dos intermetálicos $\mathrm{Ag}_{3} \mathrm{Sn}$ visíveis a olho nu.

Verificou-se também a presença de precipitados de Bi dentro das dendritas de Sn. Como já sabe, isso é consequência da diminuição da solubilidade Bi na fase $\beta$-Sn. Ao diminuir a quantidade de Sn na solução, a composição do líquido atinge a composição eutética do binário $\mathrm{Sn}-\mathrm{Bi}$, que tem o valor de $\mathrm{Sn}-58 \% \mathrm{Bi}$. Explica-se assim a fração de fase eutética. Encontra-se esse mesmo eutético em um estudo feito por Xiaowu e coautores [12]. Nesse estudo, os pesquisadores analisaram a estrutura lamelar e a microdureza durante a solidificação direcional do eutético Sn$58 \% \mathrm{Bi}$.

A Figura 4 mostra as variações dos teores de $\mathrm{Bi}$ e $\mathrm{Ag}$ ao longo do lingote $\mathrm{Sn}-40 \% \mathrm{Bi}$ $2 \% \mathrm{Ag}$. Os valores de $\mathrm{Bi}$ oscilaram em torno de $33 \%$, valor este abaixo da composição nominal. Esta ligeira oscilação pode está ligada ao fato de que a densidade do $\mathrm{Bi}$ é próxima a do $\mathrm{Sn}$. Assim, os níveis de segregação são minimizados. Além disso, a fração de fase eutética é apreciável. É sabido que o crescimento eutético envolve segregação lateral, dificultando, portanto, a rejeição de soluto para frente da interface sólido/líquido. No caso da prata, a concentração se mostrou mais alta no início do lingote, chegando ao dobro do valor nominal. Esse 
resultado está associado à alta densidade da prata $(\mathrm{Ag})$, dificultando sua segregação na interface e aumentando seu aprisionamento na fronteira sólido/líquido durante a solidificação.

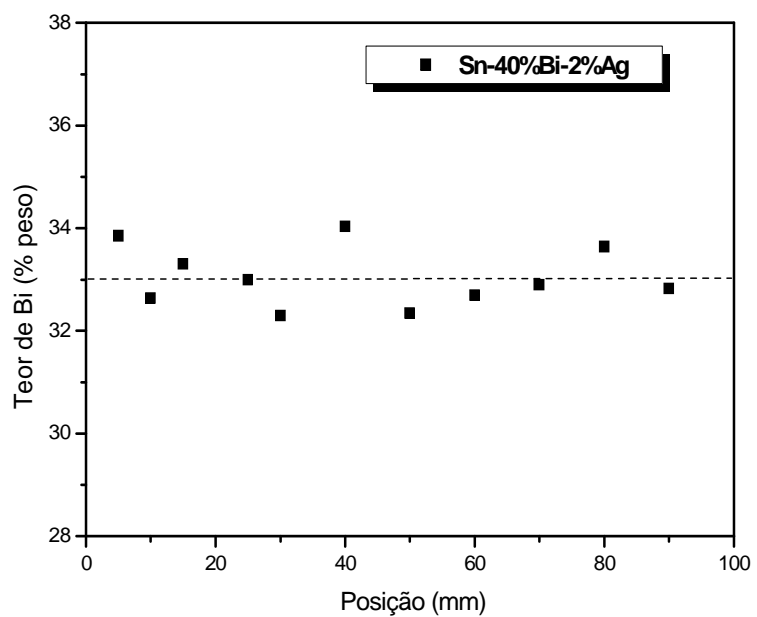

(a)

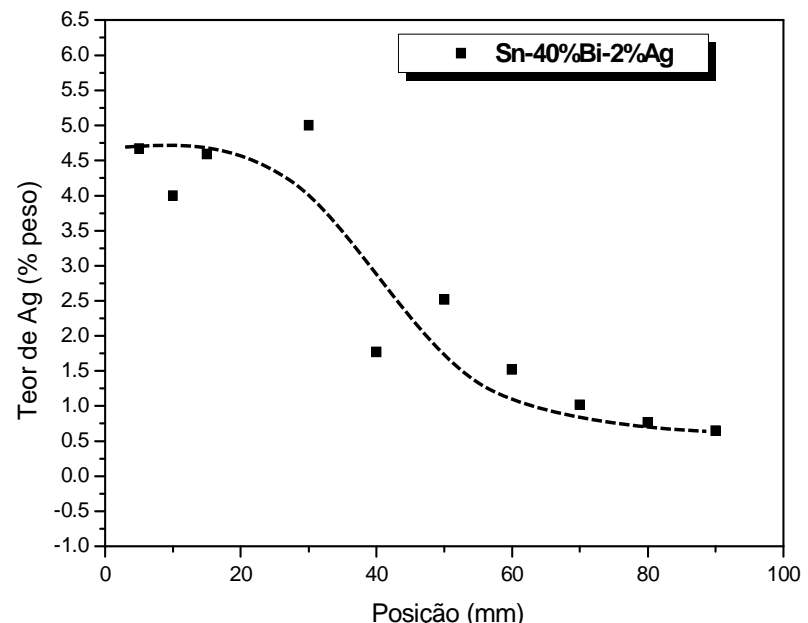

(b)

Figura 4. Perfis de macrosegregação do (a) bismuto e da (b) prata na liga Sn-40\%Bi-2\%Ag ao longo do lingote fundido.

Conforme pode ser visto no difratograma da Figura 5, as fases detectadas foram $\mathrm{Sn}$ puro, Bi puro e o composto intermetálico $\mathrm{Ag}_{3} \mathrm{Sn}$. Em alguns casos há sobreposição de picos. Notou-se que as posições avaliadas ao longo do lingote tiveram as intensidades dos picos variando ligeiramente entre si. He e Acoff [13] estudando ligas $\mathrm{Sn}-3,7 \% \mathrm{Ag}-\mathrm{XBi}$ apresentaram um difratograma com os mesmos picos abaixo relacionados.

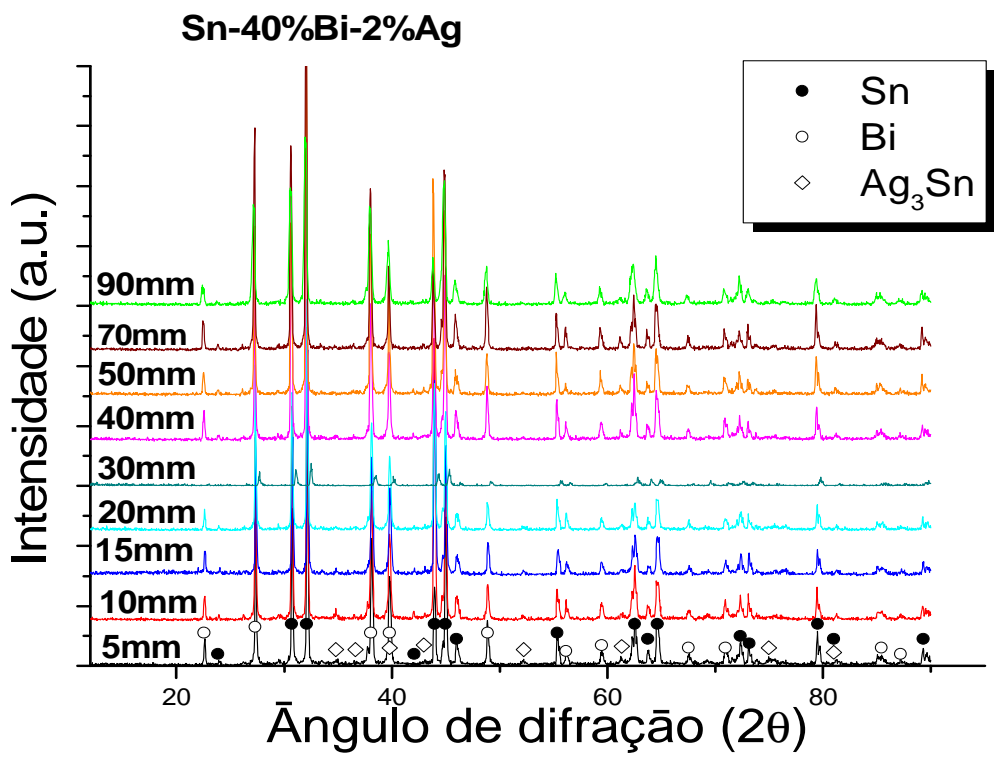

Figura 5. Difratograma ao longo de diversas posições ao longo do lingote de Sn-40\%Bi-2\%Ag.

Correlações experimentais entre espaçamentos dendríticos secundários e a velocidade da isoterma liquidus são apresentados na Figura 6. As correlações são do tipo $\lambda_{2}=A\left(V_{L}\right)^{B}$, também reportadas por outros estudos de solidificação direcional [4,6,12]. Garcia [14] estudou a liga Sn-40\%Bi solidificada direcionalmente, obtendo uma expressão $\lambda_{2}=42\left(V_{\mathrm{L}}\right)^{-2 / 3}$. Esta mesma equação foi inserida na Figura 6 para 
comparação com os resultados do presente trabalho com a liga ternária. $O$ expoente $-2 / 3$ não caracteriza a evolução de $\lambda_{2}$ para a liga ternária $\mathrm{Sn}-40 \% \mathrm{Bi}-2 \% \mathrm{Ag}$. A presença de dois solutos, além da nucleação e crescimento de partículas primárias ricas em Ag parece ter contribuído para este tipo de evolução, diferente daquelas observadas para as ligas binárias: $\mathrm{Sn}-40 \% \mathrm{Bi}$ e Sn-2\%Ag, cujo expoente $-2 / 3$ foi derivado a partir dos pontos experimentais. Acredita-se que os menores espaçamentos da liga ternária possa ser consequência da adição de $\mathrm{Ag}$ na liga binária $\mathrm{Sn}-40 \% \mathrm{Bi}$. Para velocidades inferiores a $0,3 \mathrm{~mm} / \mathrm{s}$ os valores de $\lambda_{2}$ das três ligas examinadas se aproximam de acordo com a Figura 6.

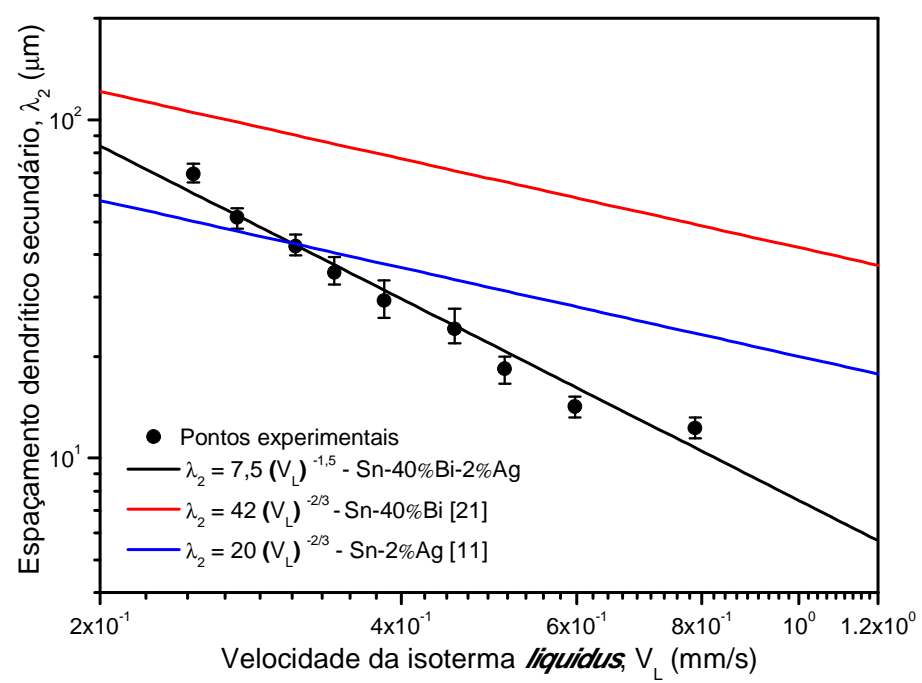

Figura 6. Variação dos espaçamentos dendríticos secundários $\left(\lambda_{2}\right)$ em função da velocidade da isoterma liquidus $\left(\mathrm{V}_{\mathrm{L}}\right)$ para a liga $\mathrm{Sn}-40 \% \mathrm{Bi}-2 \% \mathrm{Ag}$.

Os espaçamentos intercelulares e interdendríticos exercem influência sobre as propriedades mecânicas. Esse fato ocorre visto que espaçamentos menores implicam em uma distribuição mais uniforme de soluto, o que favorece uma maior resistência mecânica. Sabendo disso, foi determinada a correlação entre os espaçamentos interdendríticos secundários e os valores médios de microdureza Vickers. Uma lei experimental tipo Hall-Petch foi inserida na Figura 7. Notou-se uma ligeira diminuição dos valores de dureza em relação ao aumento do $\lambda_{2}$, visto que menores valores de $\lambda_{2}$ aumentam a propriedade de dureza em função da melhor distribuição do eutético, que é mais refinado nestas condições. 


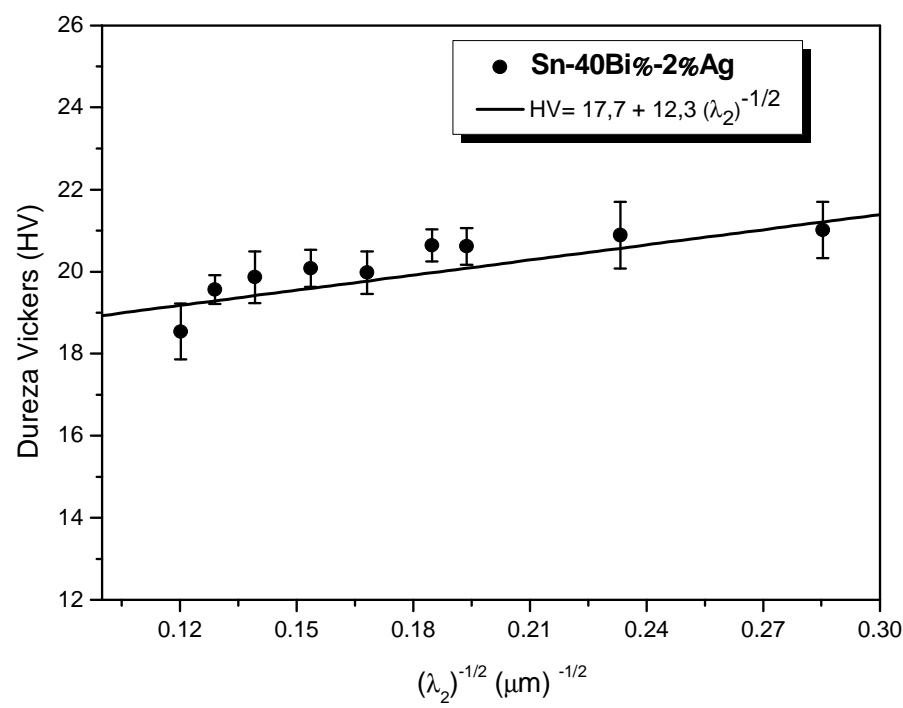

Figura 7. Variação dos espaçamentos dendríticos secundários $\left(\lambda_{2}\right)$ em função dos valores de microdureza ao longo do lingote da liga $\mathrm{Sn}-40 \% \mathrm{Bi}-2 \% \mathrm{Ag}$.

\section{CONCLUSÃO}

- A microestrutura da liga $\mathrm{Sn}-40 \% \mathrm{Bi}-2 \% \mathrm{Ag}$ é constituída de dendritas ricas em $\mathrm{Sn}$ com precipitados de Bi rodeadas de um eutético complexo lamelar ( $\mathrm{Sn+Bi}$ ), além de partículas primárias do intermetálico $\mathrm{Ag}_{3} \mathrm{Sn}$. As fases foram confirmadas pelas análises de DRX;

- Os parâmetros térmicos ( $\left.\dot{T}_{L}, V_{L}, G_{L}\right)$ decresceram devido ao aumento das resistências térmicas criadas durante a solidificação do lingote $\mathrm{Sn}-\mathrm{Bi}-\mathrm{Ag}$, o que permitiu uma ampla variação destes: $\dot{T}_{L} \rightarrow 0,1$ a $10,0^{\circ} \mathrm{C} / \mathrm{s}, V_{L} \rightarrow 0,2$ a $0,9 \mathrm{~mm} / \mathrm{s}$ e $G_{L}$ $\rightarrow 0,4$ a $11,0{ }^{\circ} \mathrm{C} / \mathrm{mm}$. O teor de bismuto não variou significativamente, enquanto que a prata variou positivamente e de forma efetiva ao longo do lingote Sn-Bi-Ag. Isto pode ser comprovado pela presença assiva de partículas ricas em Ag nas posições iniciais do lingote;

- Foi detectado o surgimento de braços dendríticos terciários a partir da posição $15 \mathrm{~mm}$, correspondendo a uma taxa de resfriamento em torno de $1,4^{\circ} \mathrm{C} / \mathrm{s}$. De um modo geral, a escala da microestrutura da liga $\mathrm{Sn}$-Bi-Ag se apresentou mais refinada em comparação com os sistemas binários $\mathrm{Sn}-0,7 \% \mathrm{Cu}$ e $\mathrm{Sn}-40 \% \mathrm{Bi}$. Os valores de microdureza Vickers diminuíram ligeiramente em função do crescimento de $\lambda_{1}, \lambda_{2} \mathrm{e}$ $\lambda_{3}$. Nessas condições, o eutético $\mathrm{Sn}$-Bi é mais grosseiro e mais heterogêneo, o que parece decrementar a propriedade de dureza.

\section{Agradecimentos}

Os autores agradecem o apoio financeiro concedido pela Fundação de Amparo á Pesquisa do Estado de São Paulo - FAPESP, com os projetos 2014/04386-3, 2013/13030-5 e 2013/08259-3.

\section{REFERÊNCIAS}

1 Abtew M, Selvaduray G. Lead-free Solders in Microelectronics. Materials Science and Engineering. 2000; 27(5-6): 95-141.

$2 \mathrm{Ma} \mathrm{H}$, Suhling JC. A review of mechanical properties of lead-free solders for electronic packaging. Journal Materials Science. 2009; 44(5): 1141-1158 
3 McCormack M, Chen HS, Kammlott GW, Jin S. Significantly improved mechanical properties of Bi-Sn solder alloys by Ag-doping. Journal of Electronic Materials. 1997; 26(8): 954-958.

4 Rosa DM, Spinelli JE, Ferreira IL, Garcia A. Cellular/dendritic transition and microstructure evolution during transient directional solidification of $\mathrm{Pb}$-Sb alloys. Metallurgical and Materials Transactions A. 2008; 39(9): 2161-2174.

5 Canté MV, Spinelli JE; Cheung N, Garcia A. The Correlation Between Dendritic Microstructure and Mechanical Properties of Directionally Solidified Hypoeutectic Al-Ni Alloys. Metallurgical Materials Internacional. 2010; 16(1): 39-49.

6 Gündüz M; Çadirli, E. Directional solidification of aluminium-copper alloys. Materials Science and Engineering A. 2002; 327(2): 167-185.

7 McCartney DG, Hunt JD. Measurements of cells and primary dendrite arm spacing in directionally solidified aluminium alloys. Acta Metallurgica. 1981; 29(11): 1851-1863.

8 Garcia A. Solidificação: Fundamentos e Aplicações. Campinas: Editora da Unicamp; 2007.

9 Density - Wikipedia, the free encyclopedia [página da internet]. São Paulo: ABM, 2015 [acesso em 11 mar. 2015]. Disponível em: http://en.wikipedia.org/wiki/Density

10 Garcia LR, Osório WR, Garcia A.The effect of cooling rate on the dendritic spacing and morphology of Ag3Sn intermetallic particles of a Sn-Ag solder alloy. Materials in Engineering. 2011; 32(5): 3008-3012.

11 Pang JHL, Xu L, Shi XQ, Zhou W, Ngoh SL. Intermetallic growth studies on Sn- Ag-Cu lead-free solder joints. Journal of Electronic Materials. 2004; 33(10): 1219-1225.

12 Xiaowu H, Ke L., Fanrong A. Research on lamellar structure and micro-hardness of directionally solidified Sn-58Bi eutectic alloy. China Foundry. 2012; 9(4): 360-365.

13 He M, Acoff VL. Effect of reflow and thermal aging on the microstructure and microhardness of Sn-3.7Ag-xBi solder alloys. Journal of Eletronic Materials. 2006; 35(12):2098-2106.

14 Garcia LR. Análise da evolução microestrutural e de propriedades mecânicas de ligas Sn-Ag e Sn-Bi para soldagem e recobrimento de superfícies [tese de doutorado] Campinas: Universidade Estadual de Campinas; 2012. 